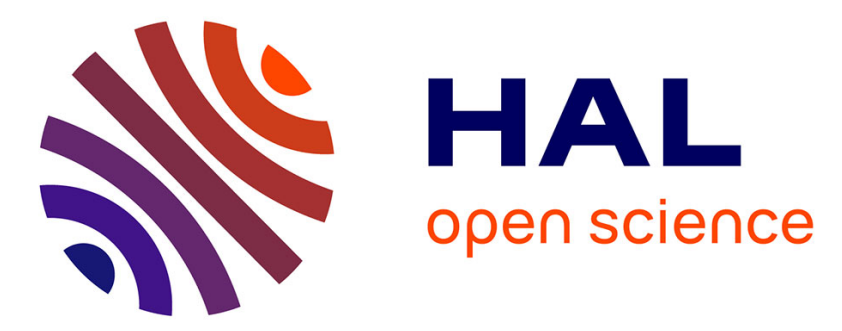

\title{
Acoustic resonator based on periodically poled lithium niobate ridge
}

Fabien Henrot, Florent Bassignot, Blandine Guichardaz, Gwenn Ulliac, Emilie Courjon, Jean-Yves Rauch, Thomas Baron, Sylvain Ballandras

\section{To cite this version:}

Fabien Henrot, Florent Bassignot, Blandine Guichardaz, Gwenn Ulliac, Emilie Courjon, et al.. Acoustic resonator based on periodically poled lithium niobate ridge. IEEE Transactions on Ultrasonics, Ferroelectrics and Frequency Control, 2013, 60, pp.1556-1563. 10.1109/TUFFC.2013.2735 . hal00931718

\section{HAL Id: hal-00931718 \\ https://hal.science/hal-00931718}

Submitted on 5 May 2021

HAL is a multi-disciplinary open access archive for the deposit and dissemination of scientific research documents, whether they are published or not. The documents may come from teaching and research institutions in France or abroad, or from public or private research centers.
L'archive ouverte pluridisciplinaire HAL, est destinée au dépôt et à la diffusion de documents scientifiques de niveau recherche, publiés ou non, émanant des établissements d'enseignement et de recherche français ou étrangers, des laboratoires publics ou privés. 


\title{
Acoustic Resonator Based on Periodically Poled Lithium Niobate Ridge
}

\author{
Fabien Henrot, Florent Bassignot, Blandine Guichardaz, Gwenn Ulliac, Emilie Courjon, \\ Jean-Yves Rauch, Thomas Baron, and Sylvain Ballandras
}

\begin{abstract}
The constant improvement of industrial needs to face modern telecommunication challenges leads to the development of novel transducer principles as alternatives to SAW and BAW solutions. The main technological limits of SAW (short-circuit between electrodes) and BAW (precise thickness control) solutions can be overcome by a new kind of transducer based on periodically poled ferroelectric substrate. The approach proposed in this paper exploits a ridge structure combined with a periodically poled transducer (PPT), allowing for the excitation of highly coupled modes unlike previously published results on planar PPTs. High-aspect-ratio ridges showing micrometer dimensions are achieved by dicing PPT plates with a diamond-tipped saw. An adapted metallization is achieved to excite acoustic modes exhibiting electromechanical coupling in excess of $15 \%$ with phase velocities up to $10000 \mathrm{~m} \cdot \mathrm{s}^{-1}$. Theoretical predictions show that these figures may reach values up to $20 \%$ and $18000 \mathrm{~m} \cdot \mathrm{s}^{-1}$, respectively, using an appropriate design.
\end{abstract}

\section{INTRODUCTION}

$\mathrm{M}$ OST RF passive components, such as resonators and filters, are based on SAW technologies with interdigitated transducers (IDTs). This mature technology particularly addresses RF filter demands, with a yearly production of billions of pieces. Although effective and massively produced, SAW devices based on IDTs are limited by various factors when addressing the demand for frequency operation in excess of $2.5 \mathrm{GHz}$. Among these limitations, the nature of the mode and single-crystal acoustic properties do not allow for the excitation of surface waves with phase velocity much higher than $6 \mathrm{~km} \cdot \mathrm{s}^{-1}$ (leaky-longitudinal SAW on lithium niobate, $\mathrm{LiNbO}_{3}[1]$ ). Also, currently implemented industrial technologies for SAW devices provide electrode width in the vicinity of $0.3 \mu \mathrm{m}$. Such lithography resolution requires strict quality control of IDT finger definition to avoid any risk of shortcircuit between electrodes (preventing the correct operation of the resulting SAW device).

Many efforts have been made to address these issues, along with various principles and structures (RF microelectromechanical systems (RF MEMS) such as film bulk acoustic resonators, solidly mounted resonators, and a population of locally vibrating parts excited using electrostatic forces [2]). An approach has been identified as

The authors are with the Franche-Comté Electronique, Mécanique, Thermique et Optique-Sciences et Technologies (FEMTO-ST) Institute, Besançon, France (e-mail: Fabien.henrot@femto-st.fr). an attractive combination of bulk and surface wave transducer characteristics, using ferroelectric polarization to generate an interference grating for acoustic waves. The so-called periodically poled transducer (PPT) is actually taking advantage of a planar definition of the acoustic period (similarly to SAWs) and a bulk propagation, allowing for various kinds of mode and acoustic propagation to be exploited. It consists of a periodic polarization of ferroelectric materials such as single-crystal $\mathrm{LiNbO}_{3}$, combined with plane electrodes exciting and detecting waves propagating in the bulk of the material [3], providing an alternative to IDTs exhibiting remarkable properties. As shown in [3], assuming a given grating period, the frequency of a PPT is twice that of an IDT, because PPTs naturally operate on a second-harmonic regime, as explained in Fig. 1. This kind of PPT was successfully manufactured, as shown in [3] and [4], but the best experimental electromechanical coupling remained smaller than $1 \%$. More specifically, in [4], a periodically poled lithium niobate (PPLN) wafer was stacked between two silicon wafers to guide acoustic waves and to reduce the temperature coefficient of frequency [4], allowing for a transducer working from $65 \mathrm{MHz}$ to $1.3 \mathrm{GHz}$ with a $\mathrm{TCF}$ of $-50 \mathrm{ppm} \cdot \mathrm{K}^{-1}$, i.e., half the TCF of a PPT on pure $\mathrm{LiNbO}_{3}$ plates.

Because this new device only allows for the excitation/ detection of acoustic modes with an electromechanical coupling reaching $1 \%$ in the best case for $\mathrm{LiNbO}_{3}$ and lithium tantalate $\left(\mathrm{LiTaO}_{3}\right)$, they are not very appropriate for modern $\mathrm{RF}$ filter challenges requiring electromechanical coupling up to $10 \%$ or even more. However, these two materials are known to allow for much more coupled mode excitation, particularly concerning bulk waves. Even if advanced transducer combinations provide advantageous conditions for wave trapping [4], PPTs based on this basic architecture are not capable of overcoming such coupling values. (Lead zirconate titanate (PZT) was found to be theoretically capable of larger mode coupling, but it is poorly suited to RF requirements because of excessive intrinsic acoustic losses [5].) To overcome this limitation, various electrode configurations and excitation conditions have been investigated, resulting in a new device architecture based on a high-aspect-ratio structure (ridge) which allows for coupling higher than 20\%. This kind of structure is used for optical waveguides $([6]-[8])$ allowing development of new devices requiring highly guided light.

Using a homemade simulation tool [9], different device designs have been studied to determine the best configurations. Ridges are achieved by dicing trenches into $\mathrm{LiNbO}_{3}$ periodically poled wafers [10]. The sides of the ridges are 

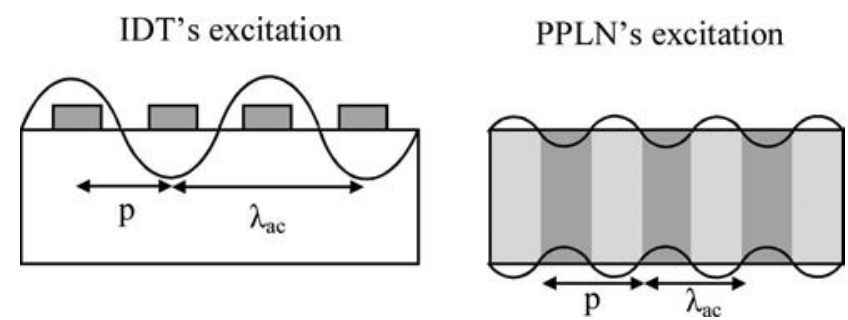

Fig. 1. Exitation principle for (left) interdigitated transducer and (right) periodically poled lithium niobate.

polished during the saw dicing, yielding wave confinement. Electrodes then are deposited on the ridge sides using gold or aluminum sputtering. The characterization of such transducers then is achieved by using a network analyzer. The agreement between theory and experiment has been checked to confirm theoretical predictions, assessing the efficiency of the developed concept and the associated analysis.

Ridge-based PPT structures are first studied using our simulation tool and the selected configurations are presented. Device manufacturing then is detailed in the next section of the paper. The obtained devices are finally characterized to emphasize the advantages of these ridgebased PPTs.

\section{PRINCIPLE AND ANALYsis}

This section exposes the working principle of PPT from wave generation to the determination of structures compliant with telecommunication requirements. Device simulation is described to emphasize its advantages compared with common RF transducers [4].

\section{A. Transducer Principle}

According to PPT principles, the new transducer is based on a periodically poled material. The most adapted crystal orientation of ferroelectric materials used for such an application must be used to periodically reverse the native polarity. Therefore, Z-cut (Z-axis pointed outward from the plate, also defined along the IEEE Std-176 standard on piezoelectric materials as (ZX) or $(\mathrm{YXl}) / 90^{\circ}$ cut [11]) $\mathrm{LiNbO}_{3}$ and $\mathrm{LiTaO}_{3}$ are the most favorable materials for this purpose. As shown in previous work [3], the $\mathrm{Z}+/$ Z- polarity alternation allows for constructive interference of acoustic waves when exciting the structure using electrodes deposited on the top and bottom sides of the plate. This former PPT has been manufactured and characterized in a previous work [3] using such an excitation principle (ZX), and more complicated transducer structures have also been derived from this initial work [4]. However, such a structure does not allow for reaching coupling factors larger than $1 \%$, which is far from meeting modern RF filter requirements. The leading idea of the present work consists of testing a new configuration

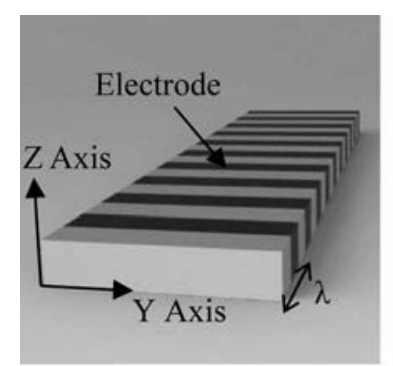

(a)

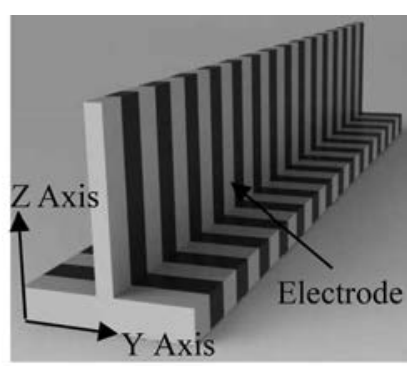

(b)
Fig. 2. (a) Previous and (b) new transducer design.

in which the excitation is achieved on the (YX) surfaces of the previously described periodically poled plates, which yields significantly larger coupling coefficients. Furthermore, this transduction approach has been shown to be capable of exciting modes exhibiting equivalent phase velocities in excess of $18 \mathrm{~km} \cdot \mathrm{s}^{-1}$, naturally yielding highfrequency operation. Fig. 2 illustrates the previous and the new configurations.

The newly designed PPT, however, requires high-aspect-ratio structures to benefit from the aforementioned characteristics of the mode. Therefore, technological investigations have been initiated to manufacture such ridges. The most appropriate solution consists of dicing periodically poled lithium niobate wafers with a high-precision saw, as further explained. The electrodes then are deposited on both sides of the PPT by sputtering to excite and detect the acoustic waves. Such high-aspect-ratio structures promote the guidance of acoustic waves along the ridge. The experimental device responses do confirm the expected properties (high velocity and electromechanical coupling compared with standard SAW devices), as discussed in Section IV.

\section{B. Analysis}

Simulations using the finite element method (FEM) are often used to explore different configurations of a system to determine the most effective ones. Different types of wave excitations in periodically poled lithium niobate are then compared for given transducer structures. In previous works [3], [4], transducers were excited through Z faces and electromechanical coupling factors of about $1 \%$ were obtained. Simulations showed that a Y-side excitation could yield coupling coefficients in excess of $20 \%$. Therefore, transducers excited on $\mathrm{Y}$ faces have been simulated to determine the optimal design (width and period). A homemade simulation software, based on finite element and boundary element methods, has been developed to study this kind of device [9]. Fig. 2 shows the 2-D mesh of one PPT period allowing for the characterization of the transducer properties derived from the so-called harmonic admittance [9]. The mesh density is very important because the more elements there are; the more precise (but time-consuming) is the simulation. A good agreement between precision and simulation time was found with the 


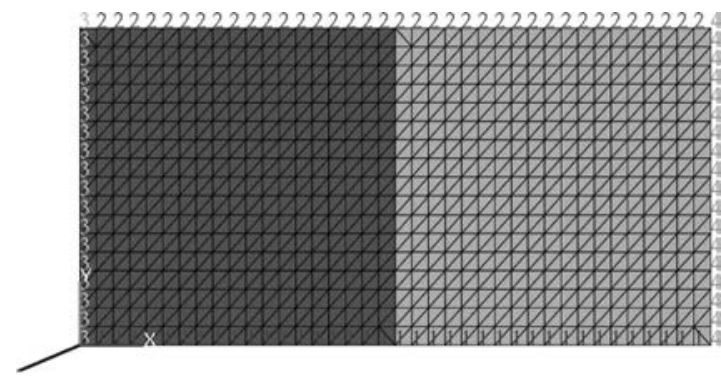

Fig. 3. 2-D mesh of one periodically poled transducer (PPT) period used for simulation.

structure shown in Fig. 2, containing 1296 elements. The adequacy between simulation and the real case will be checked to confirm the accuracy of our simulation.

The structure is periodically repeated along the $\mathrm{x}$-axis using appropriate boundary conditions (applied on references 3 and 4 in Fig. 3) to simulate an infinite transducer, and the $\mathrm{Y}$ sides are used to apply the electrical potential (on references 1 and 2 in Fig. 3). Dark and clear domains (Fig. 3), respectively, correspond to (ZX) and (ZXl) $/ 180^{\circ}$ crystallographic orientations which represent non-inverted and inverted domains. Each domain is $300 \mu \mathrm{m}$ in width, yielding a $600-\mu \mathrm{m}$ periodic structure, which is the poling period corresponding to experimentally achieved devices. The simulated configuration corresponds to an infinitely deep and infinitely long ridge and it emphasizes two interesting modes: longitudinal and a shear modes. These two modes are shown in Fig. 4(a).

The simulation results are given in Fig. 4(b), allowing for calculation of the coupling coefficient of the two distinct modes for this configuration at 16.8 and $10.3 \mathrm{MHz}$. The method for coupling estimation is given by

$$
k^{2}=1-\left(\frac{f_{\mathrm{r}}}{f_{\mathrm{a}}}\right)^{2},
$$

where $k^{2}$ is the electromechanical coupling, $f_{\mathrm{r}}$ is the resonance frequency, and $f_{\mathrm{a}}$ is the anti-resonance frequency

The electromechanical coupling of both modes depends on the width/period ratio because the device is naturally dispersive, and our simulation software allows for determining the optimal ratio for promoting the coupling factor, as shown in Figs. 5(a) and 5(b).

The best electromechanical couplings are reached for $w / \lambda$ ratios of 0.4 and 0.2 for longitudinal and shear modes, respectively. Another advantage of this configuration is the high equivalent phase velocities of these modes, which depend on $w / \lambda$ as shown in Fig. 4. Phase velocities can reach 18000 and $6500 \mathrm{~m} \cdot \mathrm{s}^{-1}$ for shear and longitudinal modes, respectively. Phase velocity is related to frequency by

$$
\lambda=\frac{c}{\nu},
$$

where $\lambda$ is the poling period, $c$ is the wave velocity, and $\nu$ is the frequency. The resonance frequencies of the two modes are also tracked in Fig. 6 to highlight the highest mode wave velocities and the modes' closeness.

Such electromechanical coupling and wave velocities could allow the device to be used in the telecommunication field as a wideband filter element. Once the optimal design (electrodes on Y sides, high-aspect-ratio structure, $w / \lambda$ ratio of 0.2 or 0.4 ) has been determined and critical parameters (poling periodicity, dicing width) found, a process is developed for manufacturing the corresponding device.

\section{Transducer Manufacturing}

The fabrication of the new device based on PPLN ridge is described in this section. First, poling methods [1], [12] are focused and detailed to understand the domain inversion phenomenon. Then, two dicing methods are described to illustrate saw capabilities for manufacturing high-aspect-ratio structures.

\section{A. Description of the Process Flow-Chart}

For this process, a 500- $\mu$ m-thick Z-cut lithium niobate wafer was used. First, a photo-lithography process is

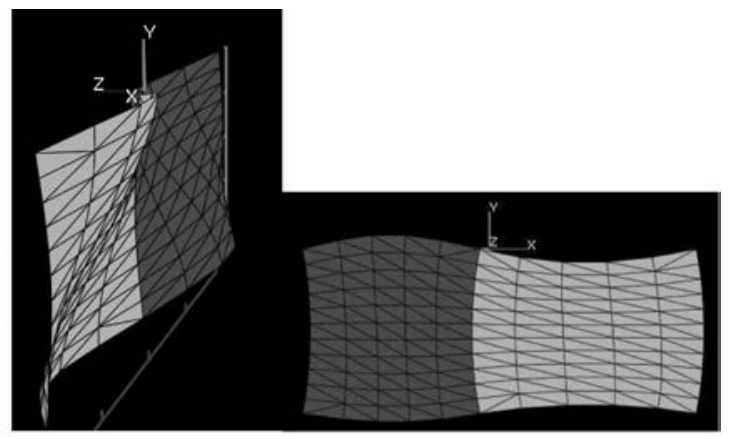

(a)

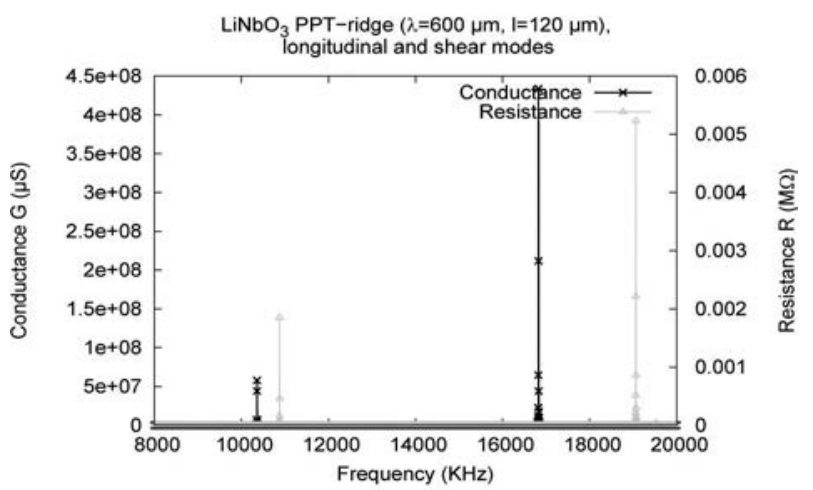

(b)

Fig. 4. (a) Simulation results for (left) shear mode and (right) longitudinal mode. (b) Harmonic admittance showing the two modes' contributions. 


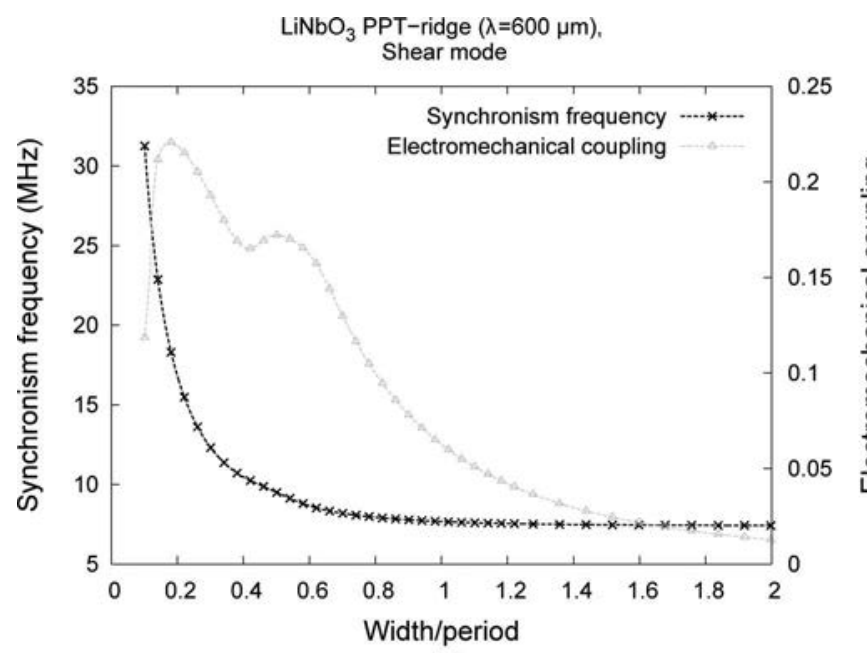

(a)

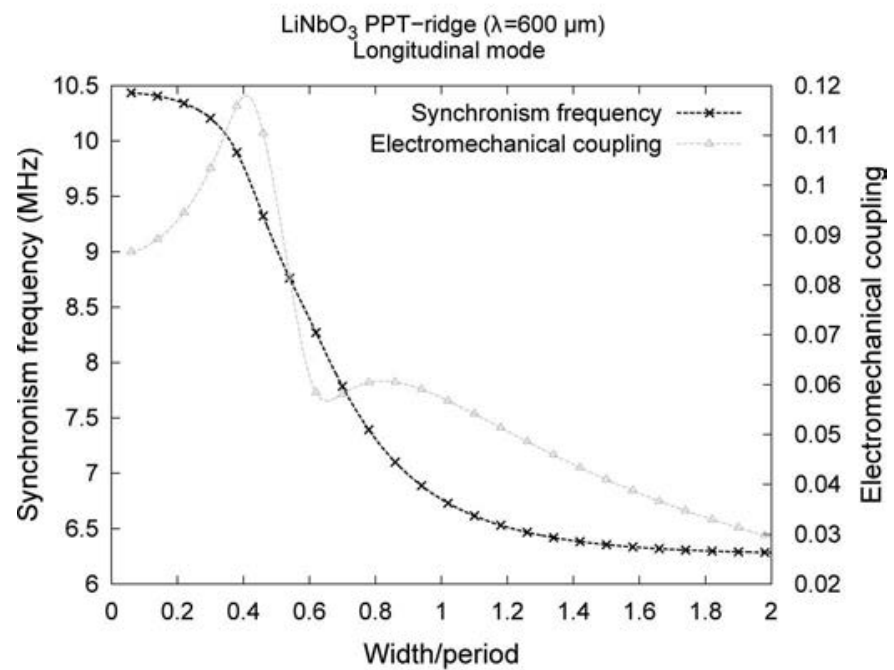

(b)

Fig. 5. Synchronicity frequencies and electromechanical coupling factors versus width/period ratio for (a) longitudinal mode and (b) shear mode.

achieved to pattern the shape of future inverted domains [Figs. 7(a) and 7(b)]. Some tests have been made to compare different photoresists (resolution, electrical insulation), showing that Shipley S1828 resist (Rohm and Haas, Philadelphia, PA) is the best solution for our application, combining good insulation and precision. Lithium niobate then is wedged between two seals to introduce an electrolyte without leaks. A mechanical system allows maintenance of the wafer and seals [Fig. 7(c)]. An electric field higher than the coercive field of lithium niobate $(21 \mathrm{kV} /$ $\mathrm{cm}$ ) is applied between the two sides of the wafer. The photo-resist layer is used as an electrical insulator. Areas covered by the photoresist are thus not inverted [Fig. 7(d)]. After the poling step, the wafer is coated with a photoresist overlay and diced to fabricate a ridge-shaped transducer. Saw cuts are made along the x-axis over depths of 100 to $500 \mu \mathrm{m}$ [Fig. 7(e)]. Gold or aluminum electrodes are then sputtered onto the device walls and patterned by lift-off. The electrode deposition method depends on the

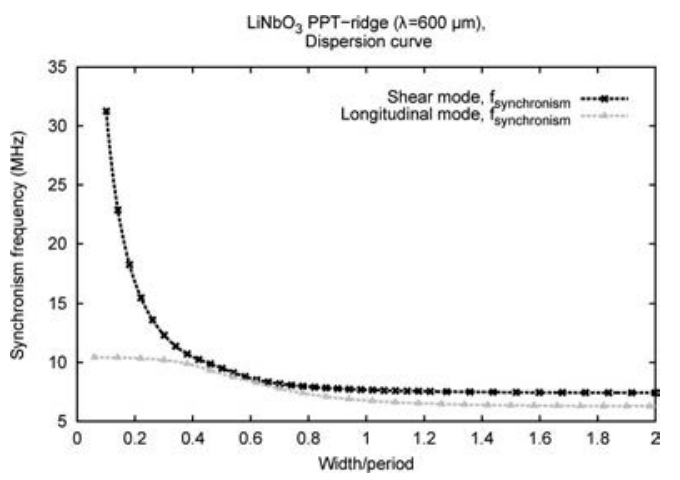

Fig. 6. Resonance frequencies versus width/period ratio for both longitudinal and shear modes.

saw cut depth. For that purpose, the device can be tilted by few degrees to reach ridge bottoms [Fig. $7(\mathrm{f})]$.

\section{B. Poling}

The poling step allows for periodic material polarity inversion by applying an electric field between the $\mathrm{Z}+$ and $\mathrm{Z}$ - faces surpassing the coercive field of the mate-

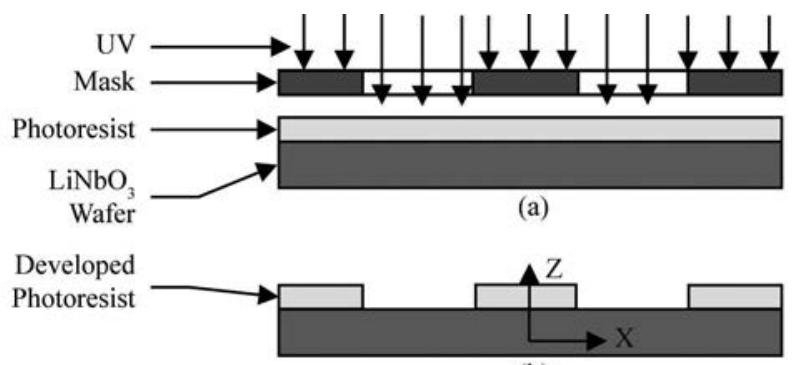

(b)

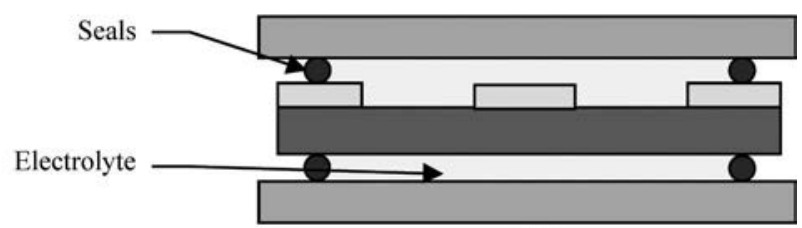

(c)

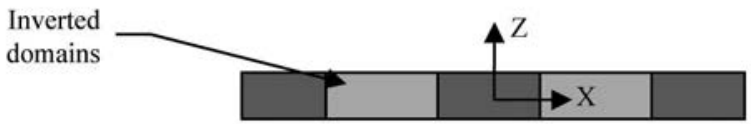

(d)

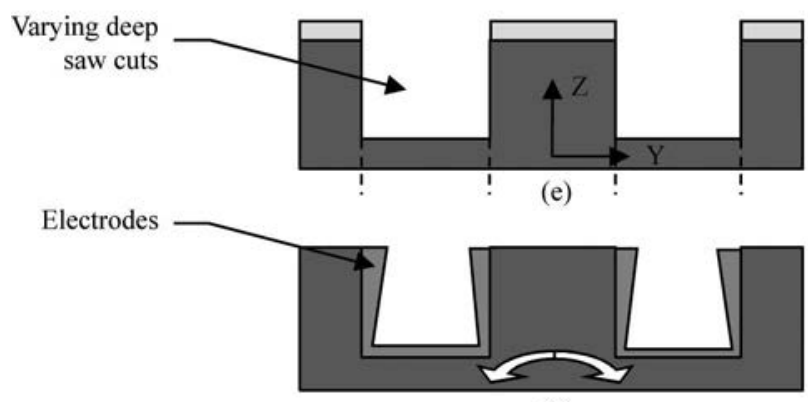

(f)

Fig. 7. Process steps for lithium niobate periodically poled transducer (PPT)-ridge fabrication. 


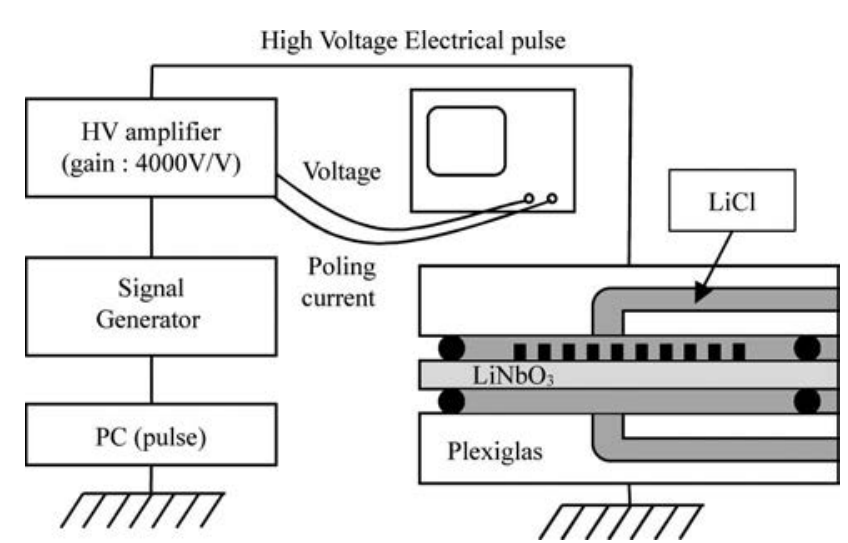

Fig. 8. Schematic diagram of the poling bench used to fabricate periodically poled ferroelectric plates.

rial. The poling bench mainly consists of a high-voltage amplifier used to submit the ferroelectric wafer to such an electric field, yielding the inversion of its native polarization. To achieve such an operation, one needs the use of optical grade Z-cut plates. A photoresist mask is achieved atop one wafer surface, which defines the poling location. A lithium chloride electrolyte is used to ensure the electrical contacts with the wafer surfaces. A dynamic poling sequence then is imposed to the wafer, progressively reaching the expected coercive field. Evidence of successful poling is obtained by measuring the current of the whole electrical system. The device is considered to be poled when recording a transient variation of this current coinciding with the application of the poling electrical field. The bench used to fabricate periodically poled $\mathrm{LiNbO}_{3}$ wafers is diagrammed in Fig. 8.

Poling is a complex process with many parameters. For further information about the poling method, the reader could refer to [3] and [12].

\section{Dicing}

The dicing step is the main technological progress for this kind of PPT-ridge. It provides the final structure of the device, and therefore determines the transducer performance, which largely depends on dicing quality and precision. This process is achieved using a Disco saw (Disco Corp., Tokyo, Japan), with a diamond blade of $400 \mu \mathrm{m}$ width and $5.2 \mathrm{~cm}$ diameter. The wafer is glued onto a photosensitive film, allowing it to be held during dicing. This film can be removed by exposing it to UV light. Two ridge-width-spaced cuts are made to obtain one ridge, as shown in Fig. 9. Parameters such as feed rate and operating speed have been determined to get the best side surface quality [13]. A photo-lithography process is first performed on the poled wafer to allow for electrode patterning. Two dicing methods have been investigated to determine the most effective one.

- The first method consists of dicing a ridge to a depth smaller than the wafer thickness $(500 \mu \mathrm{m})$, allowing

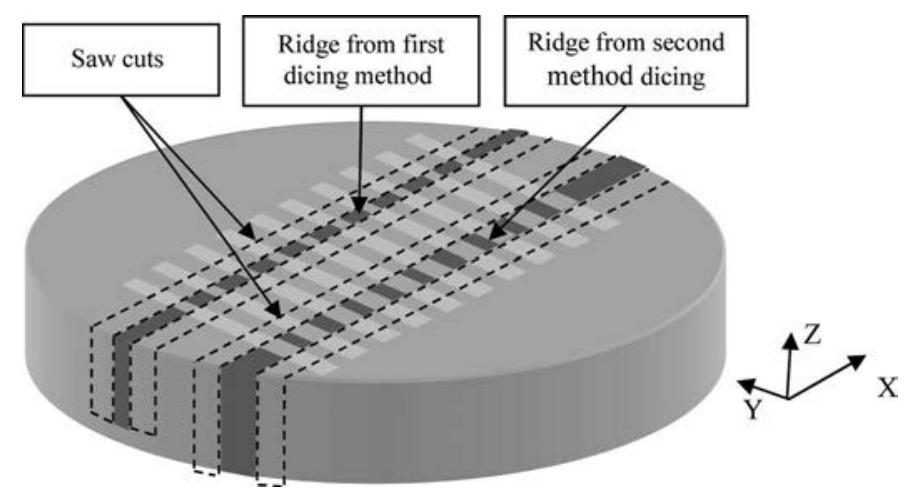

Fig. 9. Schematic diagram of wafer dicing.

the manufacture of ridges narrower than $10 \mu \mathrm{m}$ and higher than $100 \mu \mathrm{m}$. This method provides very narrow ridges, then yielding high working frequencies (above $1 \mathrm{GHz}$ ) and $w / \lambda$ ratios of 0.2 and 0.4 . An example of these ridges is shown in Fig. 10(a).

- The second method consists of dicing the entire thickness of the wafer. This method allows for manufacturing devices very close to the theoretical configurations. However, the device width can't be smaller than about $100 \mu \mathrm{m}$ because of technological limits. Consequently, the $w / \lambda$ ratio cannot be equal to 0.2 using periods smaller than $500 \mu \mathrm{m}$. Fig. 10(b) shows ridges diced through the whole depth of the wafer.

Fig. 9 illustrates the two dicing methods used to obtain structures shown in Fig. 10.

For the first method, electrodes are sputtered on ridge sides by tilting the device by a few degrees [Fig. 7(f)] A lift-off then is achieved to pattern the electrodes [Fig. $7(\mathrm{e})]$. The second method allows for a direct sputtering on ridge sides because the device can be tilted by $90^{\circ}$ within the sputtering machine.

\section{Surfaces Characterization}

The electrical response largely depends on the quality of ridge sides which are polished during dicing. The better the side quality is, the better the wave confinement is. The sawing process has been optimized (feed rate, operating speed) to obtain the best side quality. A study of ridge

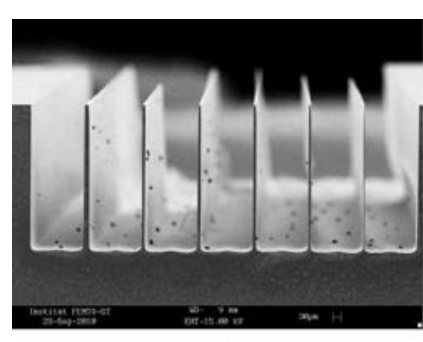

(a)

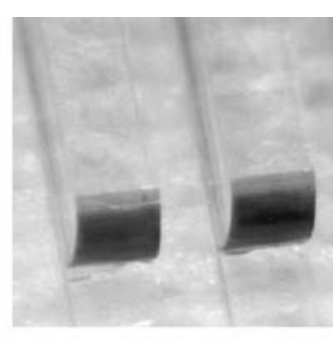

(b)
Fig. 10. Ridges achieved with (a) the first method and (b) second method. 


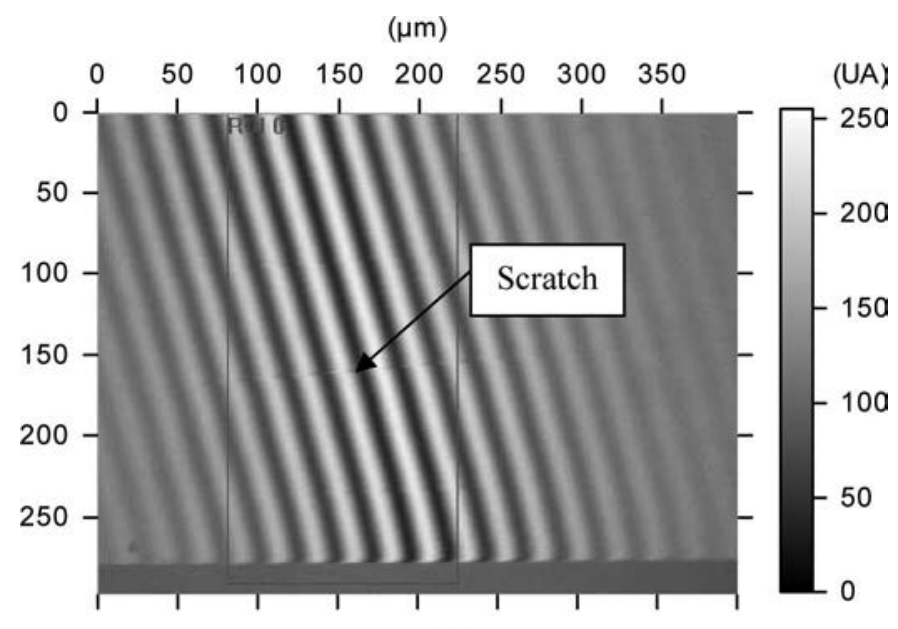

(a)

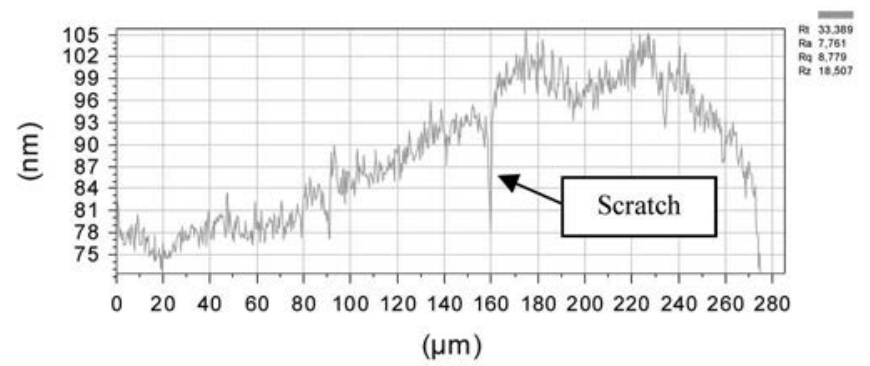

(b)

Fig. 11. (a) Image from optical profilometer and (b) measured roughness on ridge side.

shape and side roughness has been achieved to emphasize dicing quality. An optical profilometer has been used to perform these measurements. First, interferences are focalized on the studied surface and show the actual shape of the side wall [Fig. 11(a)].

The wall roughness then is measured by moving interferences on the surface. Interferences are very sensitive to depth variations and enable one to measure roughness with a precision better than $5 \mathrm{~nm}$. This sensitivity can be also very useful to spot defects on the device, such as the scratch shown in Fig. 11 and allows for a quick check of the sample's overall appearance. Results obtained by analyzing the device [Fig. 11(a)] are shown in Fig. 11(b). The roughness measured along the device lies between $50 \mathrm{~nm}$ and $5 \mathrm{~nm}$. The difference between these two values is related to the sawing direction. Indeed, the roughness is worse when the saw gets into the wafer because the

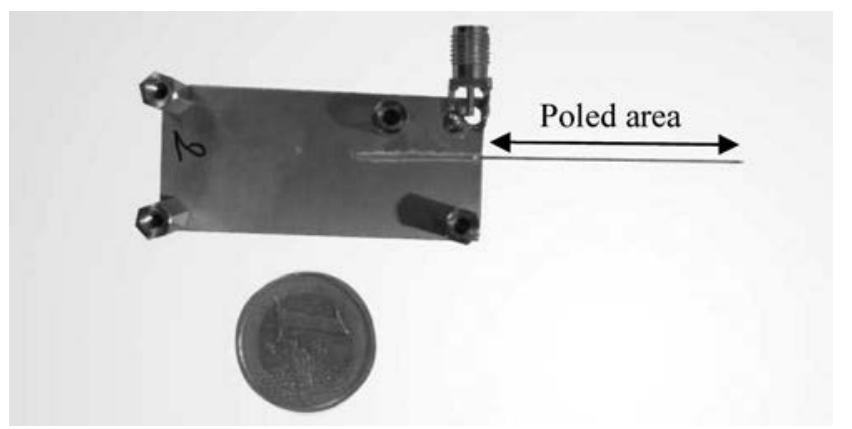

Fig. 12. As-prepared ridge-periodically poled transducer (PPT) device for characterization.

blade is better held when it is guided by the wafer. This kind of roughness allows for effective wave confinement and therefore produces highly-defined electrical responses. This result could be improved by better maintaining of the wafer on the saw chuck.

\section{E. Conclusion on Manufacturing Aspects}

Two types of devices are available, both exhibiting advantages and disadvantages.

- The first type is an embedded ridge which can reach aspect ratios higher than 20 , in accordance with the targeted $w / \lambda$ ratios. The overall quality of the device sides is better with this method compared with the second method because the ridge is held along its length. Device handling is easier in that case because it is still clamped to the wafer base. However, this embedding leads to wave leakage into the wafer, yielding unwanted insertion losses. This type of ridge can't be easily compared with simulation results because of the losses and noise caused by the embedding.

- The second type is a free-standing ridge. Therefore, its handling is quite complicated and a $w / \lambda$ ratio of 0.2 is very difficult to reach, even with a $600-\mu \mathrm{m}$ period PPLN. The sides of the ridges are crackled because the adhesive surface is drastically reduced (during dicing, this adhesive surface is reduced from $8000 \mathrm{~mm}^{2}$ to $10 \mathrm{~mm}^{2}$ ), yielding ridge motions during dicing. However, this type of device allows for a very good agreement between theoretical and experimental results. Because it corresponds to an infinite free-free ridge, the studied device model matches the actual

TABle I. Experimental (Exp.) and Theoretical (Theo.) Transducer Characterization Results.

\begin{tabular}{|c|c|c|c|c|c|c|}
\hline \multirow{2}{*}{$\begin{array}{l}\text { Length } \\
(\mathrm{cm})\end{array}$} & \multirow{2}{*}{$\begin{array}{l}\text { Depth } \\
(\mu \mathrm{m})\end{array}$} & \multirow{2}{*}{$\begin{array}{c}\text { Width/period } \\
\text { ratio } \\
(\mu \mathrm{m})\end{array}$} & \multicolumn{2}{|c|}{$\begin{array}{l}\text { Resonance } \\
\text { frequency } \\
(\mathrm{kHz})\end{array}$} & \multicolumn{2}{|c|}{$\begin{array}{c}\text { Electromechanical } \\
\text { coupling }(\%)\end{array}$} \\
\hline & & & Exp. & Theo. & Exp. & Theo. \\
\hline \multirow[t]{4}{*}{5} & 500 & 0.20 & 16665 & 16821 & 14.95 & 22.08 \\
\hline & & & 10299 & 10364 & 4.88 & 9.09 \\
\hline & & 0.33 & 11468 & 11553 & 10.94 & 18.33 \\
\hline & & & 10316 & 10093 & 8.42 & 10.76 \\
\hline
\end{tabular}



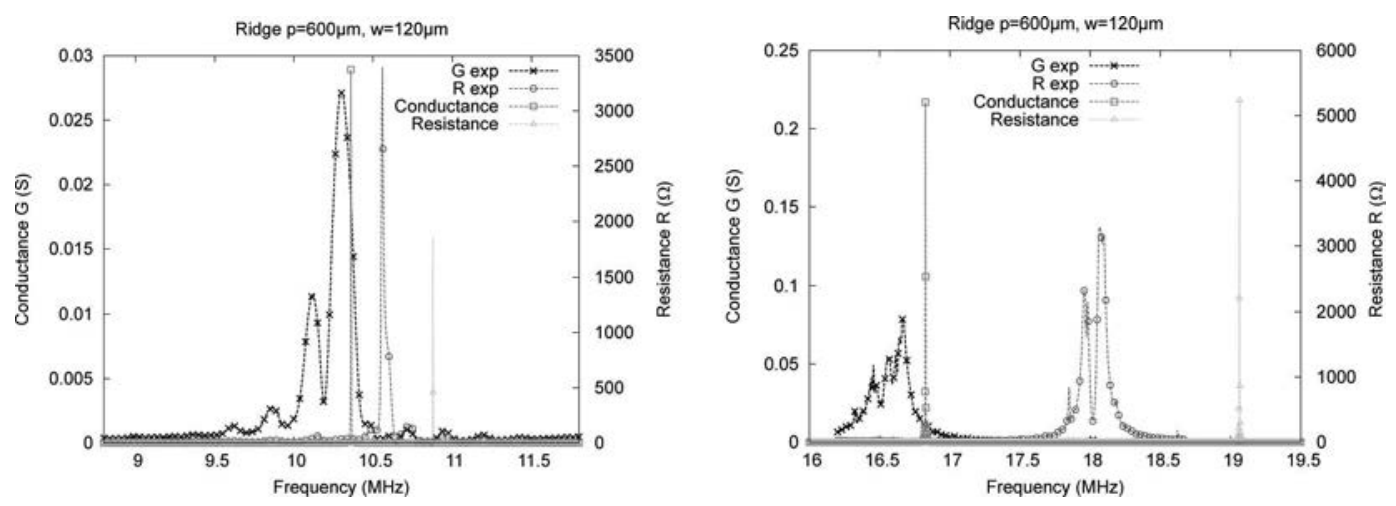

Fig. 13. Comparison between experimental (actual conductance) and theoretical (harmonic conductance) device response.

device well. Consequently, only the electrical response of the second type of device has been considered and compared with simulation results.

\section{Transducer Characterization}

Once achieved, the transducer must be characterized to compare its response to simulation results. As previously mentioned, only the second type of device is characterized here because of the simulation model and experimental setup completion. Before device's characterization, electrodes are deposited on its (YX) sides. One electrode is deposited all along the device to ensure the contact between the printed circuit board (PCB) plate and the device. Another electrode is deposited only on the poled area (see Fig. 12), allowing the excitation of this area only (avoiding parasite bulk response).

Fig. 12 shows one of the experimental devices $(\lambda=$ $600 \mu \mathrm{m}, w=120 \mu \mathrm{m})$, stuck onto a PCB with a conductive silver paste. Another device $(\lambda=600 \mu \mathrm{m}, w=$ $200 \mu \mathrm{m}$ ) has been characterized to check result coherence.

The SMA port shown in Fig. 12 is connected to the network analyzer on one side and to the device by a thin gold wire on the other side, allowing the characterization of the transducer. Device response is recorded for frequencies ranging from 1 to $30 \mathrm{MHz}$. Because of their design and dimensions, these devices were particularly suited to check the position of resonance frequencies and confirm the existence of coupling close to 20\%. Fig. 13 compares the frequency analysis of the device (effective conductance) with simulation results (harmonic conductance).

The deviation between theoretical and experimental frequencies of the two investigated modes at 10.3 and $16.8 \mathrm{MHz}$, respectively, is smaller than $1 \%$ (Fig. 8). This matching is not only explained by the adequacy between the finite element model and the actual device design but also by the accuracy of the $\mathrm{LiNbO}_{3}$ physical coefficients used in the computation [14]. The device was indeed neither poled nor excited on its stuck extremity, allowing it to be very close to a free-free model. The experimental coupling coefficient of these devices is not as high as pre- dicted, but it was found to be 20 times higher than former $\mathrm{LiNbO}_{3}$ PPTs [4]. Moreover, experimental electromechanical coupling evolution follows the theoretical prediction, as shown in Table I. The difference between theoretical and experimental couplings can be explained by the quality of the ridge sides. Because of the indented profile of the ridge, its width is not constant along its length, leading to resonant modes spreading and electromechanical coupling decreasing.

These results confirm the existence of the two predicted acoustic resonant modes close to the expected frequencies, and as a side result, the accuracy of our simulation tool. They also confirm that the coupling coefficient can reach values higher than $10 \%$, depending on the configuration.

\section{Conclusion}

A new type of structures has been successfully manufactured to allow for experimental investigation of waves excited and propagating in ridge PPTs. The obtained results are encouraging, emphasizing a very good agreement between simulation and experiment. Electromechanical coupling coefficients of about $15 \%$ have been experimentally demonstrated, according to theoretical predictions, which is a key point for the future exploitation of these devices. However, technological limits still restrict the design of ridges. Saw dicing, electrode deposition, and structure design, for example, must be improved to better control the transducer parallelism, metallization surfaces, and to reduce losses related to acoustical radiation (on-substrate ridges). Saw dicing is the key factor in obtaining a better ridge, so another dicing method will be investigated, using silicon wafer bonding to increase ridge maintenance during dicing.

\section{REFERENCES}

[1] T. Makkonen, V. P. Plessky, V. I. Grigorievski, L. Kopp, M. Solal, W. Steichen, and M. M. Salomaa, "FEM/BEM simulation and experimental study of LLSAW resonator characteristics on YZ$\mathrm{LiNbO}_{3}, "$ in IEEE Ultrasonics Symp., 2002, vol. 1, pp. 317-320.

[2] R. Ruby, "Review and comparison of bulk acoustic wave FBAR, SMR technology," in IEEE Ultrasonics Symp., 2007, pp. 1029-1040. 
[3] E. Courjon, N. Courjal, W. Daniau, G. Lengaigne, L. GauthierManuel, S. Ballandras, and J. Hauden, "Lamb wave transducers built on periodically poled Z-cut $\mathrm{LiNbO}_{3}$ wafers," J. Appl. Phys., vol. 102 , art. no. $114107,2007$.

[4] F. Bassignot, E. Courjon, G. Ulliac, S. Ballandras, J.-M. Lesage, and R. Petit, "Acoustic resonator based on periodically poled transducers: Concept and analysis," J. Appl. Phys., vol. 111, art. no. 064106, 2012.

[5] P. Muralt, J. Antifakos, M. Cantoni, R. Lanz, and F. Martin, "Is there a better material for thin film BAW applications than AlN?" in IEEE Ultrasonics Symp., 2005, vol. 1, pp. 315-320.

[6] S. W. Kwon, W. S. Yang, H. M. Lee, W. K. Kim, H.-Y. Lee, W. J. Jeong, M. K. Song, and D. H. Yoon, "The ridge waveguide fabrication with periodically poled $\mathrm{MgO}$-doped lithium niobate for green laser," Appl. Surf. Sci., vol. 254, no. 4, pp. 1101-1104, 2007.

[7] J.-H. Zhao, X.-H. Liu, Q. Huang, P. Liu, and X.-L. Wang, "Lithium niobate ridge waveguides fabricated by ion implantation followed by ion beam etching," J. Lightwave Technol., vol. 28, no. 13, pp. 1913-1916, 2010.

[8] B. Weigand, M. Stolze, F. Rubel, J. L'huillier, A. Lenhard, C. Becher, and S. Wolff, "Fabrication of ridge waveguides in $\mathrm{LiNbO}_{3}$," in Proc. Int. Symp. Applications of Ferroelectrics, held jointly with European Conf. Applications of Polar Dielectrics and Int. Symp Piezoresponse Force Microscopy and Nanoscale Phenomena in Polar Materials, 2012, pp. 1-4.
[9] S. Ballandras, P. Lardat, M. Wilm, Th. Pastureaud, A. Reinhardt, N. Champavert, W. Daniau, V. Laude, R. Armati, and G. Martin, "A mixed finite element/boundary element approach to simulate complex guided elastic wave periodic transducers," J. Appl. Phys., vol. 105, art. no. 014911, 2009.

[10] K. Suizu, Y. Suzuki, Y. Sasaki, H. Ito, and Y. Avetisyan, "Surfaceemitted terahertz-wave generation by ridged periodically poled lithium niobate and enhancement by mixing of two terahertz waves," Opt. Lett., vol. 31, no. 7, pp. 957-959, 2006.

[11] IEEE Standard on Piezoelectricity, ANSI/IEEE Std. 176, 1987.

[12] G. D. Miller, "Periodically poled lithium niobate: Modeling, fabricaiton, and nonlinear-optical performance," PhD. thesis, Dept. of Electrical Engineering, Stanford University, Stanford, CA, 1998.

[13] N. Courjal, B. Guichardaz, G. Ulliac, J.-Y. Rauch, B. Sadani, H. Lu, and M.-P. Bernal, "High aspect ratio lithium niobate ridge waveguides fabricated by optical grade dicing," J. Phys. D, vol. 44, art. no. 305101, 2011

[14] G. Kovacs, M. Anhorn, H. E. Engan, G. Visintini, and C. C. W. Ruppel, "Improved material constants for $\mathrm{LiNbO}_{3}$ and $\mathrm{LiTaO}_{3}$," in Proc. IEEE Ultrasonics Symp., 1990, pp. 435-438. 